\title{
SIMULAÇÃO DO ESCOAMENTO EM CONTRACORRENTE ÁGUA/AR EM REATORES NUCLEARES PWR UTILIZANDO CÓDIGO OPENFOAM
}

\author{
Y. MORGHI ${ }^{1, *}$, A. Z. MESQUITA ${ }^{1}$, J. PUENTE ${ }^{2}$, A. R. BALIZA ${ }^{3}$ \\ ${ }^{1}$ Centro de Desenvolvimento da Tecnologia Nuclear (CDTN / CNEN - MG), Campus da UFMG - Pampulha, \\ ${ }^{2}$ Centro Federal de Educação Tecnológica Celso Suckow da Fonseca, Campus Angra dos Reis - RJ, \\ ${ }^{3}$ Eletrobrás Termonuclear S.A. - ELETRONUCLEAR \\ ssfmorghi@gmail.com*
}

Submetido 02/10/2017 - Aceito 22/11/2018

DOI: $10.15628 /$ holos.2018.5643

\section{RESUMO}

A limitação do escoamento em contracorrente, ou inundação, é um fenômeno caracterizado pelo controle que um gás exerce no escoamento de um líquido em sentido contrário. Este efeito tem recebido atenção especial da área nuclear, devido à sua influência no comportamento termofluidodinâmico dos reatores nucleares refrigerados à água pressurizada (Pressurized Water Reactor - PWR), durante um acidente de perda de refrigerante - LOCA (Loss-of-Coolant Accident). A modelagem numérica constitui uma ferramenta fundamental para o desenvolvimento da engenharia nuclear. Este trabalho tem o popósito de demonstrar que o software de dinâmica de fluídos computacional CFD (Computational Fluid Dynamics) OpenFOAM ${ }^{\circledR}$, tem potencial para ser utilizado na modelagem do fenômeno de escoamento em contracorrente que ocorre no núcleo dos reatores PWR. Uma introdução de CFD usando OpenFOAM. O solver utilizado foi o interFoam, onde foi observado que o openFOAM apresentou resultados satisfatórios para o estudo de escoamento multifásicos.

PALAVRAS-CHAVE: OpenFOAM, interFoam, Escoamento Bifásico, Termo hidráulica, CCFL

\section{SIMULATION OF THE WATER / AIR COUNTERCURRENT FLOW IN THE PWR NUCLEAR REACTORS USING OPENFOAM CODE}

\section{ABSTRACT}

The limitation of countercurrent flow, or flooding, is a phenomenon characterized by the control that a gas exerts in the flow of a liquid in the opposite direction. This effect has received special attention from the nuclear area due to its influence on the thermofluid dynamics behavior of Pressurized Water Reactor (PWR) during a Loss of Coolant Accident (LOCA). Numerical modeling is a fundamental tool for the development of nuclear engineering. This work has the purpose to
\end{abstract}

demonstrate that the Computational Fluid Dynamics (CFD) software OpenFOAM ${ }^{\circledR}$ has the potential to be used in the modeling of the countercurrent flow phenomenon that occurs in the core of PWR reactors. An introduction of CFD using OpenFOAM. The solver used was interFoam, where it was observed that openFOAM presented satisfactory results for the multiphase flow study.

KEYWORDS: OpenFOAM, interFoam, Multiphase, Hydraulic engineering, CCFL 


\section{INTRODUÇÃO}

A limitação do escoamento em contracorrente - CCFL (Countercurrent Flow Limitation) de água e ar é de grande importância no campo da análise de segurança dos reatores nucleares. A possível ocorrência de CCFL na perna quente de um reator PWR, durante um acidente de perda de refrigerante (Loss of Coolant Accident-LOCA), ou também acidentes de pequena ruptura SBLOCA (Small-Break Loss-of-Coolant Accident) é de especial interesse para a investigação de segurança nuclear (Navarro, 2005).

O CCFL, ou inundação, representa uma condição na qual um fluxo de gás estabelece um controle sobre o fluxo de líquido na direção oposta. Tendo em vista a grande importância de entender este fenômeno na indústria nuclear, várias instalações experimentais no mundo foram construídas, inclusive no Brasil, no CDTN existe o circuito água-ar (CAA), conforme mostrado na figura 1. (Navarro, 2005; Valée et al., 2007; Deendarliando, 2011; Al Issa, 2014).

Tendo em vista que o CCFL é um fenômeno que ocorre devido à interação de dois fluidos - ar e água, atualmente também tem sido utilizada a fluidodinâmica computacional para analisálo. A fluidodinâmica computacional - CFD (Computational Fluid Dynamics) é um ramo da dinâmica de fluidos que fornece um meio eficaz com custo e tempo baixos, para simulação de escoamentos reais para solução numérica das equações que regem um fenômeno. 0 desenvolvimento da CFD moderno começou no início dos anos 1960 a partir do desenvolvimento dos computadores e atualmente conta com o desenvolvimento de vários softwares que são capazes de criação de geometrias complexas, geração de malhas, solução de equações de conservação e fornecem dados de saída que mostram o fenômeno acontencendo na própria geometria além de dados numéricos em gráficos e tabelas. (Valée et al., 2007; Utanohara et al., 2012).

Neste artigo, as simulações foram feitas em uma tubulação horizontal, ou seja, na mesma direção da tubulação do Sistema de Refrigeração de um Reator PWR. A geometria foi desenvolvida utilizando SolidWorks e a malha foi desevolvida utilizando SALOME para simular a interação entre água e ar no interior desta tubulação horizontal e o CFD utilizado foi o OpenFoam.

\section{O INICIO DA CCFL}

A limitação do escoamento em contracorrente (CCFL) ocorre quando o líquido e o gás fluem em direções opostas. Se o fluxo de massa do gás aumenta muito, o fluxo de líquido é interrompido e, em seguida, a água será transportada pelo gás, podendo ser parcialmente ou totalmente empurrada na direção oposta.

O início da CCFL corresponde à condição limitante em que as taxas de fluxo do gás ou do líquido podem aumentar sem alterar o padrão de escoamento e limitar a taxa de fluxo de líquido (Navarro, 2005). Este é o ponto de estabilidade de limitação do fluxo de contracorrente indicado pela taxa máxima de fluxo de massa de ar, em que a taxa de fluxo de massa de água que flui para baixo é igual à taxa de fluxo de massa de água de entrada (Deendarliando et al., 2011). Esta 
condição limitante é também conhecida como início de inundações, podendo acontecer em geometria vertical ou horizontal.

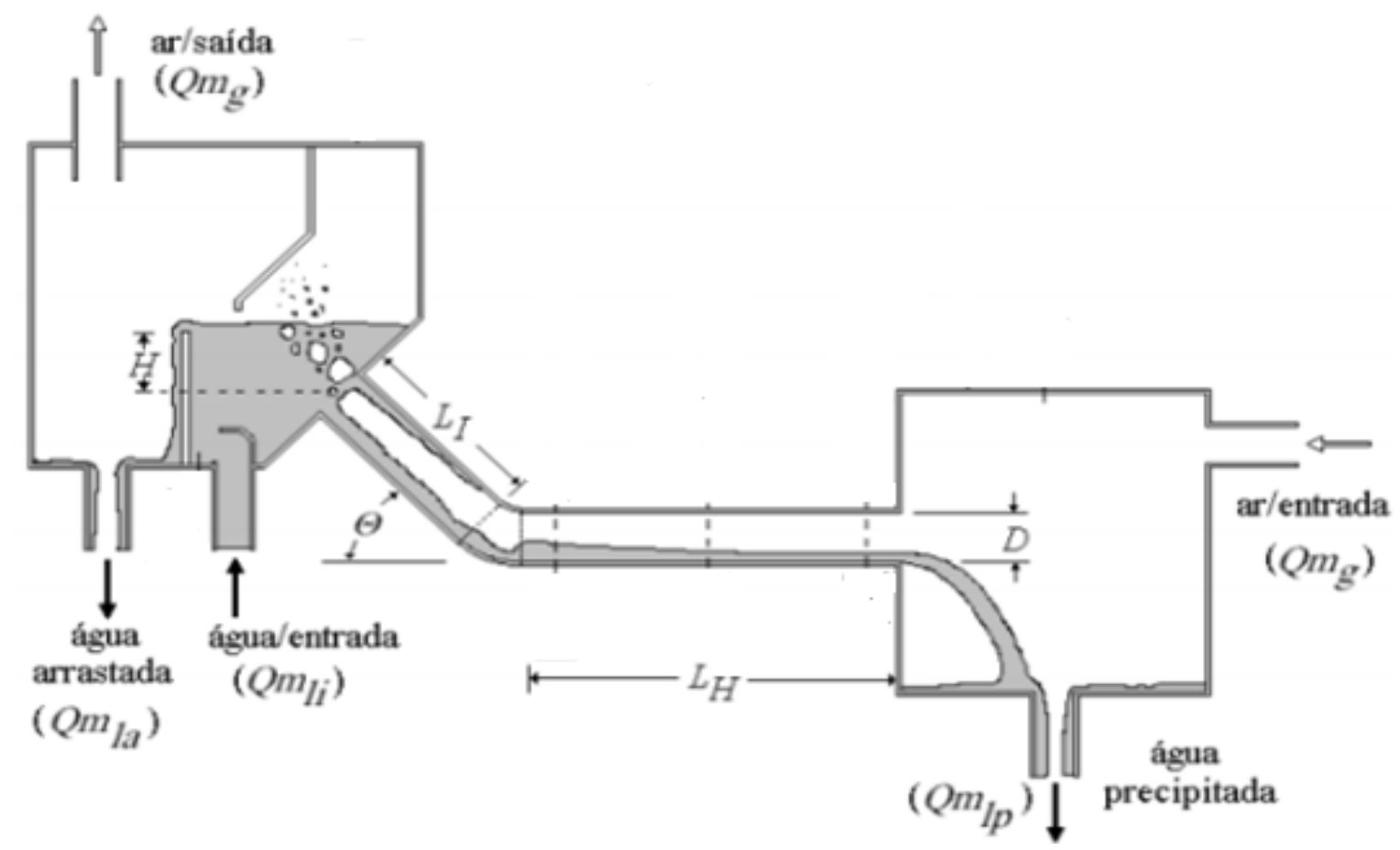

Figura 1: Seção de testes do Circuito Água-ar (CAA) - CDTN/MG (Navarro, 2005).

Escoamentos em contracorrente de água e gás são de grande importância no campo da análise de segurança dos reatores nucleares (Navarro, 2005). O CCFL pode ocorrer na pernaquente de um reator à água pressurizada (PWR), durante um acidente de perda de refrigerante, LOCA ou SBLOCA e em caso de perda do sistema de remoção residual de calor.

Algumas investigações experimentais de CCFL foram realizadas conforme desenho esquemático da Figura 1, em uma geometria constituída de um tubo horizontal ligado a um tubo ascendente inclinado (Al Issa, 2014; Navarro, 2005).

Na geometria da perna quente uma estratificação suave é estabelecida para velocidades superficiais baixas de líquidos e gases. Conforme a velocidade do gás aumenta, perturbações começam a aparecer na interface. Pequenas ondas se iniciam e crescem. Instabilidades, disturbios hidráulicos, crescimento de ondas, arrastamento de gotas, e movimento caótico da interface, levando ao desenvolvimento do CCFL, conforme mostra a Figura 2.

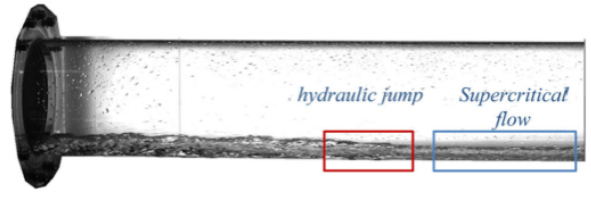

a )

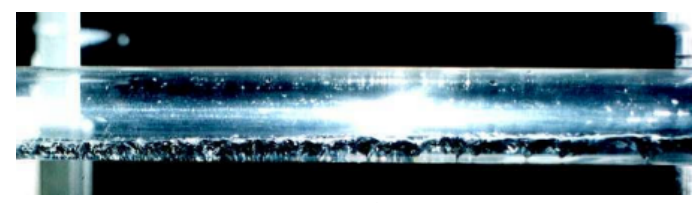

b)

Figura2: (a) A aparência do salto hidráulico perto da saída de água, (b) Inundação do escoamento em CCFL. 


\section{MODELO CFD PARA ESCOAMENTO MULTIFÁSICO}

A modelagem multifásica faz parte do modelo matemático dentro do processo de realização de uma simulação de escoamento de fluidos. Todo fluxo de fluidos que consiste em mais de uma fase é um fluxo multifásico, este pode ser, de gás/líquido, líquido/líquido, gás/sólido ou líquido/sólido.

Em escoamento de gás/líquido os fluxos de cada fase podem coexistir em cada ponto do espaço-tempo e um novo parâmetro aparece que é a fração de vazio, $\alpha$, conforme equação 1 . Por exemplo, se há um fluido de duas fases que consiste de líquido e gás, e o invólucro é formado por apenas a fase gasosa:

$\alpha=\frac{\text { Volume de Gás }}{\text { Volume Total }}$

Os códigos CFD são baseados em algoritmos numéricos e consistem em três elementos principais: pré-processamento, processamento e pós-processamento.

No passo de pré-processamento tem-se: definição da geometria, geração de malha, definição de propriedades do fluido, as propriedades turbulência e as condições iniciais e de contorno. Vale ressaltar que, a precisão de uma solução CFD é afetada pelo número de células na malha.

No processamento ou agente de resolução, as variáveis de fluxo desconhecidas são aproximadas por meio de funções simples, em seguida, estas aproximações são introduzidas nas equações governantes e elas são discretizadas mais tarde. Finalmente as equações algébricas são resolvidas. O OpenFOAM usa volume finito para definir a maneira pela qual as variáveis de fluxo são aproximadas e os processos de discretização. No método de volumes finitos, o algoritmo numérico segue estes passos:

- A integração das equações governantes sobre todo o volume de controle do domínio.

- Conversão das equações integrais em um sistema de equações algébricas através da discretização.

- Solução das equações algébricas.

Finalmente, tem-se o pós-processamento dos dados criados para serem analizados. Nesta etapa são visualizados a geometria, a grade, os perfis e parcelas.

Tendo em vista o fenômeno CCFL, o movimento do fluido é regido por um conjunto de equações que expressam a conservação de massa, de quantidade de movimento e de energia:

- Conservação de massa:

$\frac{\partial_{\rho}}{\partial t}+\nabla \cdot(\rho u)=0$ 
- Conservação de quantidade de movimeto:

$\frac{\partial \rho u}{\partial t}+\nabla \cdot(\rho u u)=\rho g+\nabla \cdot \sigma$

- Conservação de energia:

$\frac{\partial \rho e}{\partial t}+\nabla \cdot(\rho e u)=\rho g u+\nabla \cdot(\sigma u)-\nabla \cdot q+\rho Q$

Onde $\rho$ é a massa especifica, u é o campo de velocidade tridimensional, $\sigma$ é o tensor de tensão de corte, e é a energia específica total, a $Q$ é a fonte de energia de volume, q é o fluxo de calor e g é o vetor de aceleração da gravidade.

No solver interFoam, o método VOF (Volume de Fluido) convencional utiliza a fração de vazio como uma função indicadora (alpha no código OpenFOAMTM) para definir a parte da célula que é ocupada pelo fluido.

O transporte de $\alpha$ no tempo é expresso por uma função de advecção:

$\frac{\partial \alpha}{\partial t}+\nabla \cdot U \alpha=0$

$\alpha$ é a fração do vazio e conserva-se para o espaço e tempo. É um valor escalar entre 0 e 1 , em que 0 significa fase gasosa e 1 fase líquida.

$\alpha=0: \quad$ fase gasosa

$\alpha=1$ : fase líquida

$0<\alpha<1$ : a célula que contém uma interface.

\section{MODELAGEM COMPUTACIONAL E RESULTADOS}

Os casos em OpenFOAM têm sempre a mesma estrutura. Inicialmente dentro da pasta de cada caso 3 outras pastas são encontradas: 0 /, constante e / system /.

A pasta 0 / contém todas as condições iniciais e de contorno para os diferentes campos de fluxo.

Dentro da pasta constante / dados a partir da geometria e a malha são encontrados, bem como as propriedades de transporte e propriedades de turbulência e especificações.

Finalmente, dentro da pasta sistema / diretório todos os dados sobre as informações esquemáticas e o passo de tempo numérico são encontrados. Isto pode ser verificado na Figura 3. 


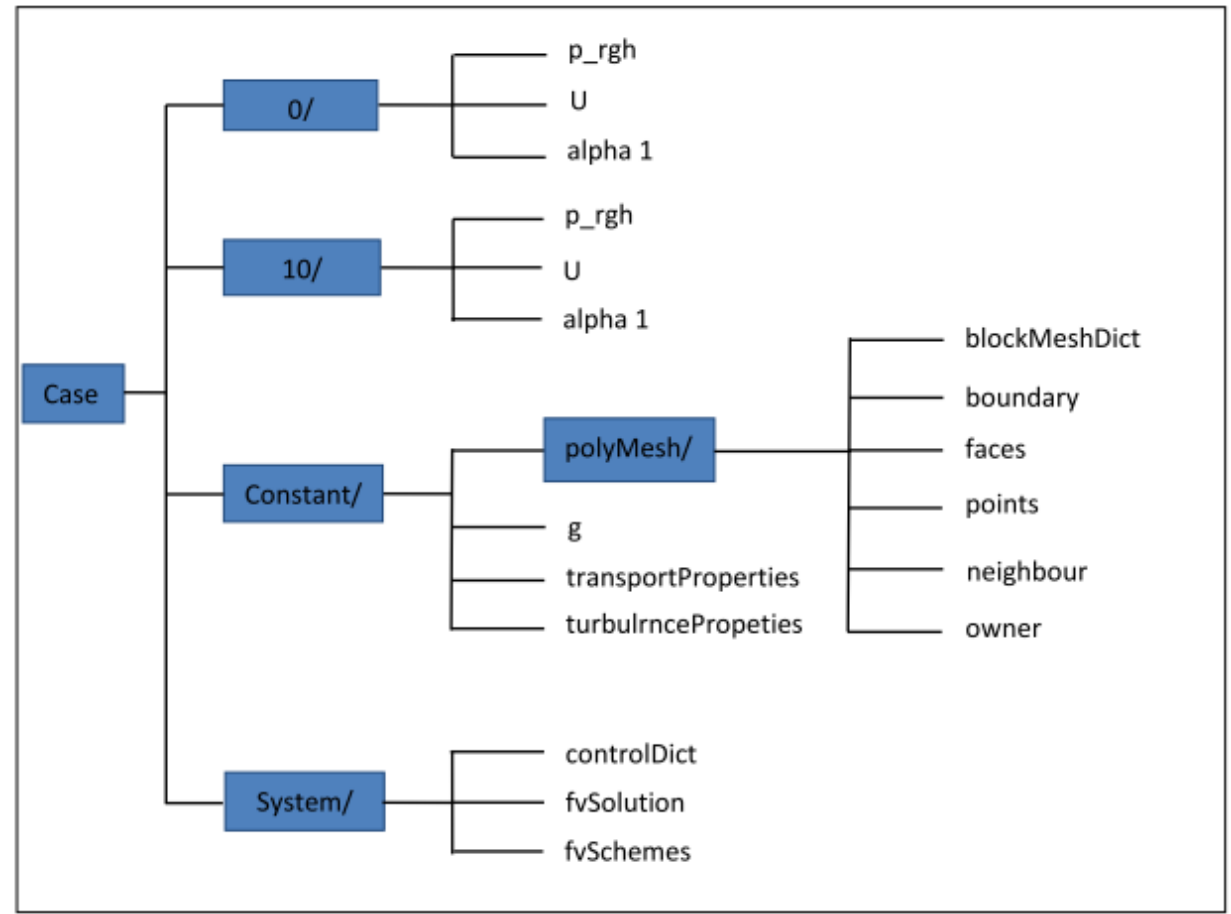

Figura 3: Exemplo de um caso OpenFOAM com o tempo final de 10 segundos

\subsection{Pré-processamento}

\subsubsection{Geração da geometria e da malha}

A geometria foi desenvolvida utilizando SolidWorks 2015 e a malha foi desenvolvida utilizando SALOME V7-8-0 para simular a interação entre água e ar no interior de uma tubulação horizontal.

Os dados da malha são:

Pontos:

Pontos internos: 0

Faces: $\quad 4070$

Faces Internas: $\quad 1930$

Celulas: $\quad 1000$

Faces Por celula: 6 (hexahedra)

A qualidade da malha é essencial para o sucesso e precisão dos resultados. Se a qualidade da malha não for adequada os resultados não serão satisfatórios e uma nova malha deve ser definida. No entanto, para uma malha mais fina é necessário mais tempo e capacidade de processamento do computador.

\subsubsection{Limite e Condições Iniciais}

As faces de contorno são "inlet-water" e "inlet-air" para a entrada , "outlet-water" e "outlet-air" para as saídas e "lowerWall" para a parte inferior da parede e "topWall" para a parte superior . 
A entrada da velocidade é o valor que muda nos diversos casos que estão sob estudo. 0 valor para a entrada é definido como "zeroGradient". A saída é definido como "zeroGradient", o que significa que a velocidade normal ao fluxo de saída é zero. Para as paredes, a velocidade é também uma "fixedValue" mas é definido como 0, devido à condição sem deslizamento do fluido nas paredes. Em relação à pressão, uma vez que a velocidade é fixada nas entradas e nas paredes, a pressão dinâmica é definida como "zeroGradient", de modo que o gradiente normal às faces seja zero. A condição de saída limite para a pressão também está definida para "zeroGradient".

Como a interação de ar e água acontece em sentidos opostos, como simulado neste artigo, ocorre turbulência, sendo assim, é considerado que o valor para o número de Reynolds (Re) atinge valores acima do Re crítico, ou seja Re maior que 4000. Neste caso, os valores de k e $\varepsilon$ que são para ser usados no modelamento de turbulência também são especificados nas condições de contorno. $O$ modelo das condições de contorno que é usado neste artigo é baseado no tutorial damBreak (OpenFOAM 2.3).

As vantagens do modelo $k-\varepsilon$ são: que este é o modelo mais simples para modelos de turbulência e tem um excelente desempenho para muitos fluxos relevantes na indústria e está bem estabelecido (OpenFOAM, user guide 2016).

\subsubsection{Propriedades físicas}

As propriedades do fluido são definidas no dicionário do openFOAM "transportProperties". As propriedades da água e do ar (gás) são definidas nos sub dicionários de fase 1 e fase 2 respectivamente. As fases (como fluidos Newtonianos) têm definidas, neste caso, os valores de viscosidade e densidade, como se segue:

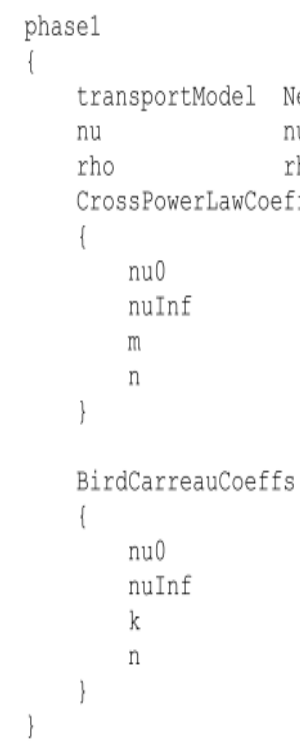

a)

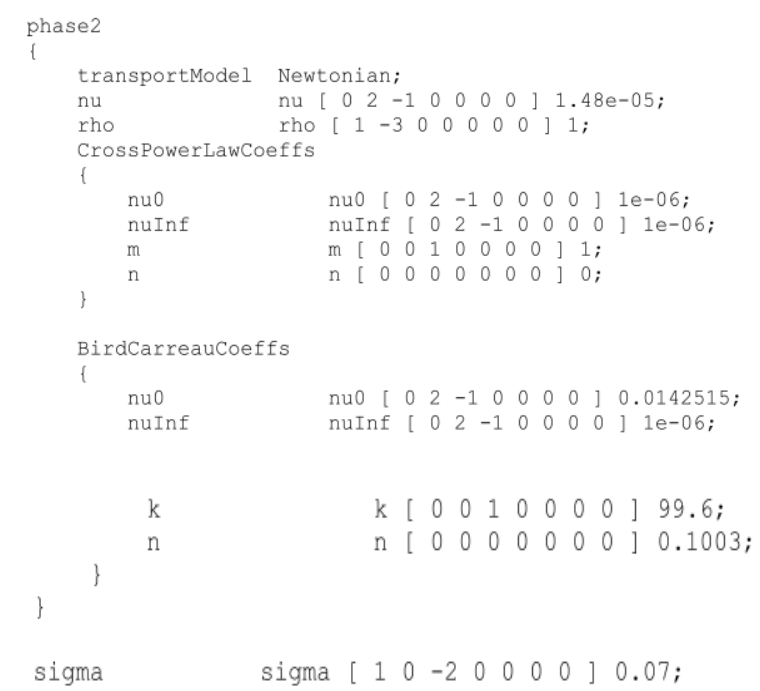

b)

Figura 4 (a) - Propriedades do fluido - fase 1, Figura 4 (b) - Propriedades do fluido - fase 2

\subsubsection{Total de Interações e Controle de Saída de dados}

O algoritmo de seguimento de superfície em interFoam é mais sensível ao número Courant do que outros modelos, por causa da importância dos valores precisos na interfase. 


$$
C_{0}=\frac{\delta \mathrm{t}|U|}{\delta \mathrm{x}}
$$

Em que $C_{0}$ é o número Courant, $\delta$ t é iteração, $|U|$, magnitude da velocidade através dessa célula, $\delta$ x é o tamanho da célula na direção de velocidade.

Para este caso o número Courant é escolhido como 0,5, a fim de alcançar a precisão temporal e estabilidade numérica ao executar o solver.

"Time steps" para este caso são definidos da seguinte forma no arquivo "controlDict":

$\begin{array}{ll}\text { application } & \text { interFoam; } \\ \text { startFrom } & \text { startTime; } \\ \text { startTime } & 0 ; \\ \text { stopAt } & \text { endTime; } \\ \text { endTime } & 10 ; \\ \text { deltaT } & 0.001 ; \\ \text { writeControl } & \text { adjustableRunTime; } \\ \text { writeinterval } & 0.05 ;\end{array}$

Figura 5 - Total de Interações e Controle de Saída de dados

\subsection{Pós-processamento}

A principal ferramenta de pós-processamento fornecido com OpenFOAM é o "Paraview", um aplicativo de visualização de código aberto. As propriedades do vetor podem ser colorido de acordo com alguma outra propriedade de fluxo, tais como a pressão (vermelho para alta pressão e azul para baixa pressão), velocidade ou temperatura. Deste modo é fácil visualizar não só a magnitude e a direção do fluxo, mas outras propriedades, bem como, visualizá-las simultaneamente. O "Paraview" é lançado por escrito no terminal "paraFoam". Velocidade, pressão e perfil de frações de vazio podem ser vistos no "Paraview".

\subsection{Resultados}

Um dos objetivos do trabalho é gerar os padrões de fluxo, que são características do escoamento multifásico que ocorrem durante um LOCA em um reator PWR, a fim de atingir estes padrões a velocidade das fases tem de ser manipulada.

Uma vez que as simulações são feitas, os resultados para diferentes velocidades de água e ar são obtidos. Os resultados são apresentados sob a forma de um traçado cruzado ou mapa com a velocidade superficial do $a r, U_{a r}$, no eixo dos $X$ e a velocidade de líquido superficial, $U_{l q}$, no eixo dos y.

Sendo assim, foi projetada uma geometria de um elemento cilíndrico de fluido incompressível que flui em uma tubulação, conforme Figura 6. 


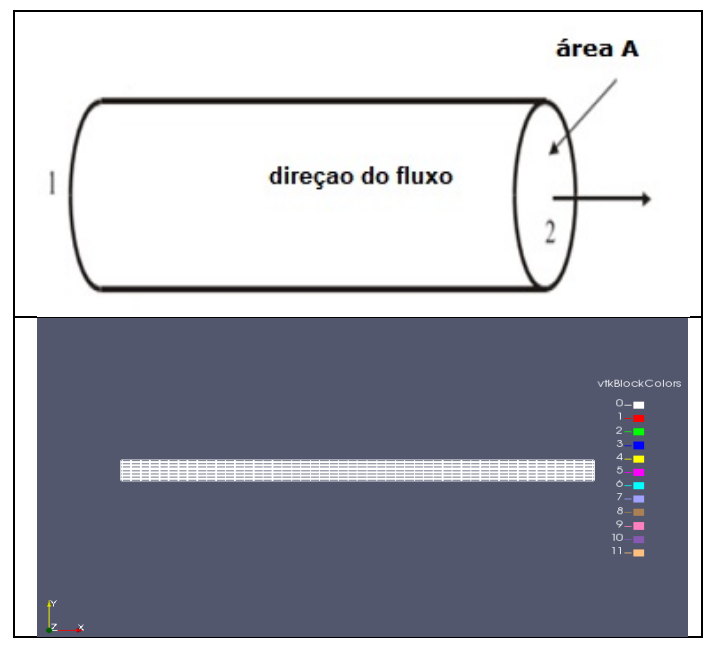

Figura 6. Elemento de fluxo de fluido numa tubulação - Geometria (SolidWork) e malha (Salome).

O primeiro caso simulado é o caso em que ambas as fases têm a mesma velocidade num canal horizontal. A velocidade é, em seguida, ajustada a $1 \mathrm{~m} / \mathrm{s}$ para o ar e para a água. Como pode ser visto na Figura 7, existe uma separação das fases e ocorre apenas o fluxo continuo. Neste estado durante todo o domínio computacional, não há mistura entre as duas fases e a interface é suave.

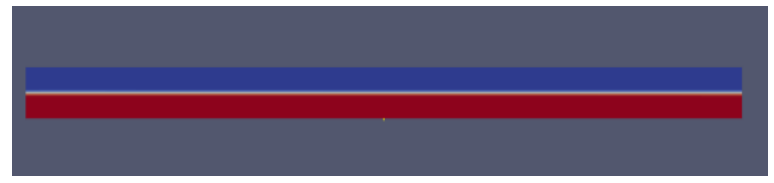

Figura 7. Fase distribuição - (OpenFoam)

A distribuição da pressão é normal, tal como pode ser visto na figura 8 , é um valor constante para cada fase e a alteração abrupta acontece na interface devido à mudança da densidade.

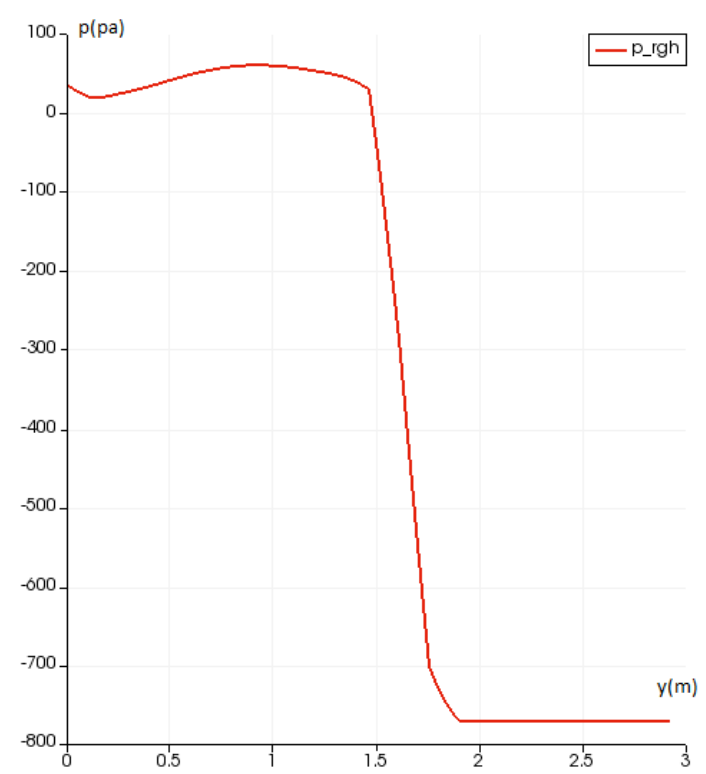

Figura 8. Distribuição de pressão - (OpenFoam) 
A fim de observar o fenômeno CCFL a velocidade de uma das duas fases tem de ser maior do que a outra. Neste segundo caso de simulação, a velocidade de água foi mantida constante, ou seja, não variou com o tempo e a de ar foi sendo variada (aumentada) com o tempo. Em baixas taxas de fluxo, são observadas bolhas na parte superior do tubo devido à flutuabilidade (Figura 9(a)). Mas, quando a velocidade de ar continua aumentando, as bolhas vão se distribuindo uniformemente no líquido (Figura 9(b)).

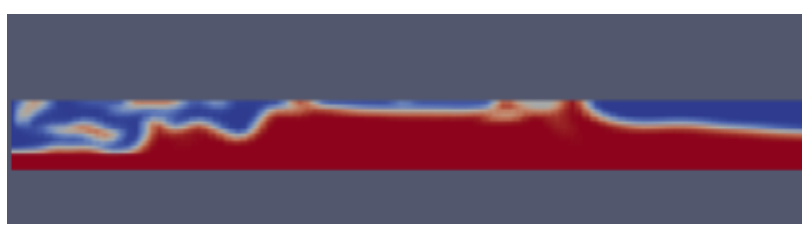

a)

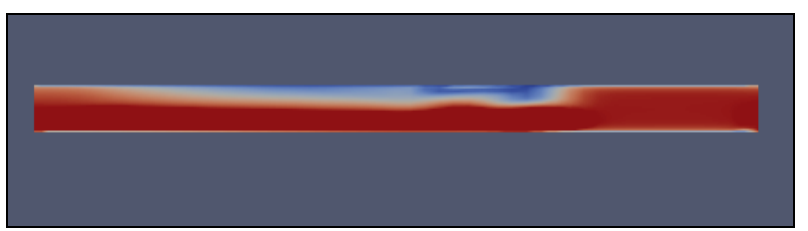

b)

Figura 9: (a) Fase distribuição em $t=1 s, \quad(b)$ Fase distribuição em $t=3 s$ (OpenFoam)

Quando a velocidade do ar é suficientemente elevada, o padrão do fluxo obtido é anular. Em teoria, o ar flui para dentro de um núcleo interno e a água forma uma película sobre a parede do canal.

Se a velocidade do ar continua aumentando, a água irá derramar a partir da camada de película e vai arrastar como gotas para o núcleo do ar. Este regime é conhecido como fluxo névoa anelar e existe um equilíbrio entre as gotículas que lançam a partir da camada líquida e as bolhas de coalescência nele. Nesta etapa, o início do processo de arraste é antecipado com o aumento da velocidade de ar.

Como acontece nos casos anteriores, a fase líquida é muito mais rápida que o ar. Como mostrado na figura 10. A água começa a colapsar no final do canal e uma onda na direção oposta aparece.

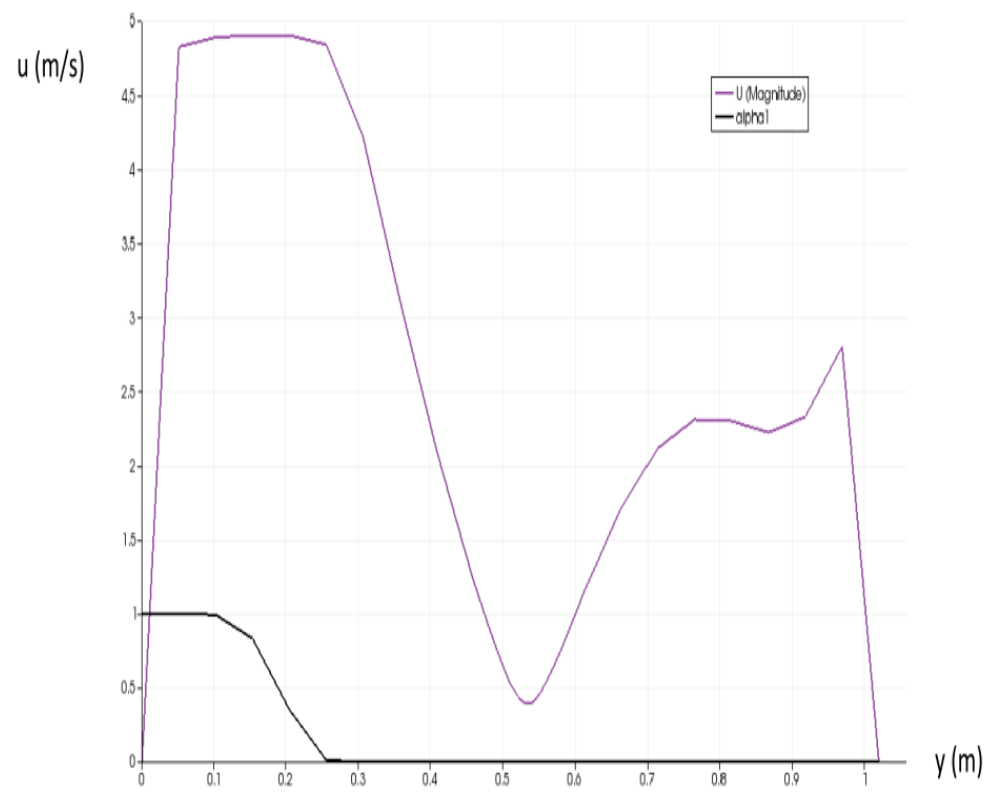

Figura 10 - Velocidade do perfil $t=4 s$ (OpenFoam) 


\section{CONCLUSÃO}

Com os resultados obtidos pôde-se verificar que o OpenFOAM apresenta um bom desempenho quando se trabalha com fluxos multifásicos simples usando o "interFoam solver".

Foram demonstradas duas simulações, onde a geometria utilizada e a direção do fluxo, são semelhantes à de uma tubulação do sistema de refrigeração de um reator de usina PWR, onde para o primeiro caso, no qual, a velocidade para ambas as fases são iguais, um padrão estratificado é obtido e no segundo caso, quando o valor de velocidade de água é mantido constante e o de ar aumenta com o tempo, pôde ser observado que o início do processo de formação de bolhas, ou seja, de turbulência, sendo observado, assim, o fenômeno CCFL.

\section{REFERÊNCIAS}

Issa, S. \& Macian, R., (2014). Experimental investigation of countercurrent flow limitation (CCFL) in large-diameter hot-leg geometry: A detailed description of CCFL mechanisms, flow patterns and high-quality HSC imaging of the interfacial structure in a 1/3.9 scale of PWR geometry. Nuclear Engineering and Design, 280, 550-563.

Utanohara, Y., Kinoshita, I., Murase,M., Dirk, L., Vallée, C,. \& Tomiyama, A., (2012). Numerical simulations for steam-water CCFL tests using the 1/3 scale rectangular channel simulating a PWR hot leg. Nuclear Engineering and Design, 249, 14-23.

Vallée, C., Seidel, T., Dirk L., Beyer, M., Prasser, H.M., Pietruske, H., Schutz, P., \& Helmar, C., (2012). Counter -current flow limitation in a model of the hot leg of a PWR-comparison between air/water and steam/water experiments. Nuclear Engineering and Design, 245, 113124.

Deendarliando, Vallée, C,. Dirk, L., Beyer, M., Pietruske, H., \& Helmar C,. (2011). Experimental study on the air/water counter-current flow limitation in a model of the hot leg of a pressurized water reactor. Nuclear Engineering and Design, 241, 3359-3372.

Vallée, C,. Hohne, T., Prasser, M., \& Suhnel, T,. (2007). Experimental investigation and CFD simulation of horizontal stratified two-phase flow phenomena. Nuclear Engineering and Design, 238, 637-646.

Navarro, M.A., (2005). Study of countercurrent flow limitation in a horizontal pipe connected to an inclined one. Nuclear Engineering and Design, 235, 1139-1148.

OpenCFD Ltd. (2016). OpenFOAM (Version 2.3), the Open Source CFD Toolbox, CFD Direct. OpenFOAM user guide. http://cfd.direct/openfoam/user-guide/ 